\title{
Giant aneurysmal bone cyst mimicking malignant tumor
}

\author{
Andrzej Borowski, Marek Drobniewski, Michał Skrzypek, Kajetan Pstrągowski, Marek A. Synder, \\ Magdalena Krasińska, Marek Synder
}

Clinic of Orthopaedics and Paediatric Orthopaedics, Medical University of Lodz, Poland

Aneurysmal bone cyst $(A B C)$ is a bone tumor which occurs mostly in young adults. Despite its benign character, it may sometimes locally turn malignant and can reach a significant size. Therefore, it is not uncommon for this disease to become similar to a malignant, destructive process and to be difficult to diagnose. We present a case of a 12-year-old girl with a rapidly growing mass in the region of the left hip joint. Imaging ( $\mathrm{X}$-ray, MRI, CT) showed a growing lesion in the proximal part of the femur that damaged the cortical layer of the bone. This suggested a presence of rhabdomyosarcoma. The lesion was removed surgically and the bone defects were filled with grafts. Two months after the surgery the patient reported pain in the area of the right groin and left knee. X-ray showed bone lesions in the right pubic bone and the left femoral bone. The biopsy showed no pathological lesions. One year after the surgery, lesions in the X-ray image of the right pubic bone and left femoral bone remitted spontaneously. Ten years after the diagnosis of the aneurysmal bone cyst no recurrence of the disease or other ailments was observed.

NOWOTWORY J Oncol 2019; 69, 2: 67-70

Key words: aneurysmal bone cyst (ABC), diagnosis, operative treatment

\section{Introduction}

Aneurysmal bone cyst $(A B C)$ is a bone tumor which occurs mostly in young adults [1]. This benign fibro-osseous lesion is considered to be a secondary vascular consequence of fibrous dysplasia or giant cell tumor and is generally found in long bones. The lesion is usually benign, however it may sometimes locally turn malignant and can grow. Unfortunately, this disease is difficult to diagnose.

\section{Case report}

We would like to present a case of a 12-year-old girl who was diagnosed with a rapidly growing tumor in the area of the left hip joint. During the first visit the patient reported that during the last 4 weeks after the exercise she felt increasing pain. X-ray showed a lesion in the proximal part of the femur (Fig. 1). It formed growing mass and damaged the cortical layer of the femur with periosteal reaction.

In turn the magnetic resonance imaging (MRI) of the left femur showed the growing masses in the metaphysis of the femur and in $1 / 3$ of the proximal femoral shaft. The damage concerned the cortical layer of the bone. A fracture was also observed in the antero-lateral part of the femur. The results of the above mentioned imaging tests suggested rhabdomyosarcoma.

Computed tomography $(\mathrm{CT})$ mostly confirmed the presence of cystic, extensive tumor with evident damage to the anterior and lateral cortical layer of femoral cortical bone (Fig. 2). However, it was only the biopsy that made it possible to diagnose an aneurysmal bone cyst (ABC) on the basis of fibrous dysplasia without any malignant features.

The patient was qualified for surgical resection of the tumor. Bone defects were filled with allogeneic cancellous bone grafts (Fig. 3).

Two months after the surgery, during the follow-up visit, the patient reported pain in the area of the right groin and left knee. Scintigraphy of the skeleton was performed (Fig. 4) and imaging studies, such as X-ray and CT scan (Fig. 5). The presence of lesions in the left femur and the right pubic bone 


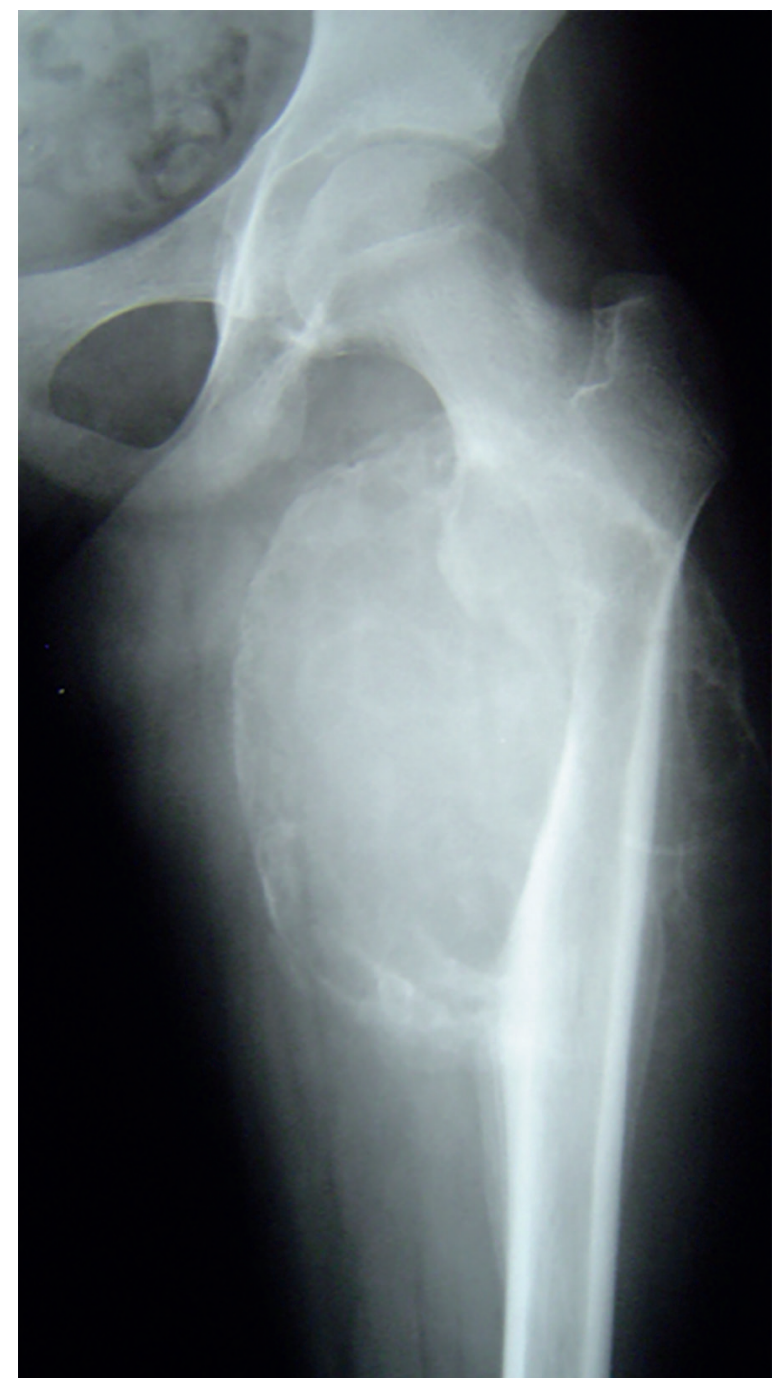

Figure 1. X-ray image in antero-posterior projection of femoral bone with visible massive, diffusing bone lesion

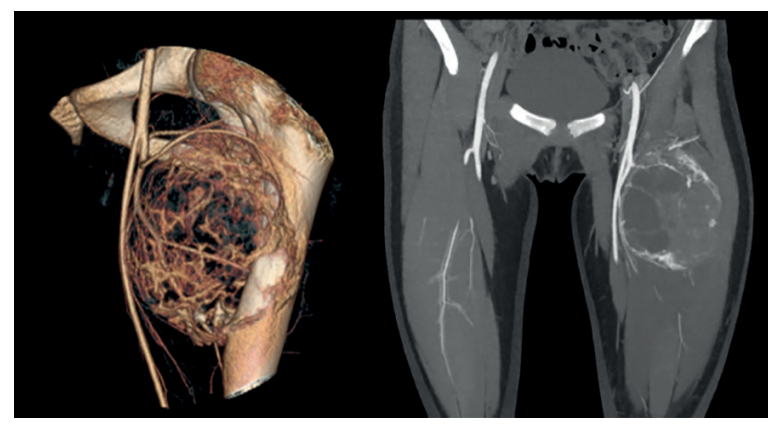

Figure 2. Image in computed tomography of the proximal part of the femur with 3D reconstruction

was observed. The thigh biopsy showed no pathological lesions. The pain disappeared spontaneously after a few days, so further diagnostics of the lesions found in scintigraphy was not continued.

The X-ray picture taken 4 months after the surgery showed a good reconstruction of bone grafts and no local recurrence. However, the periosteal reaction of the "peel-like"type was still

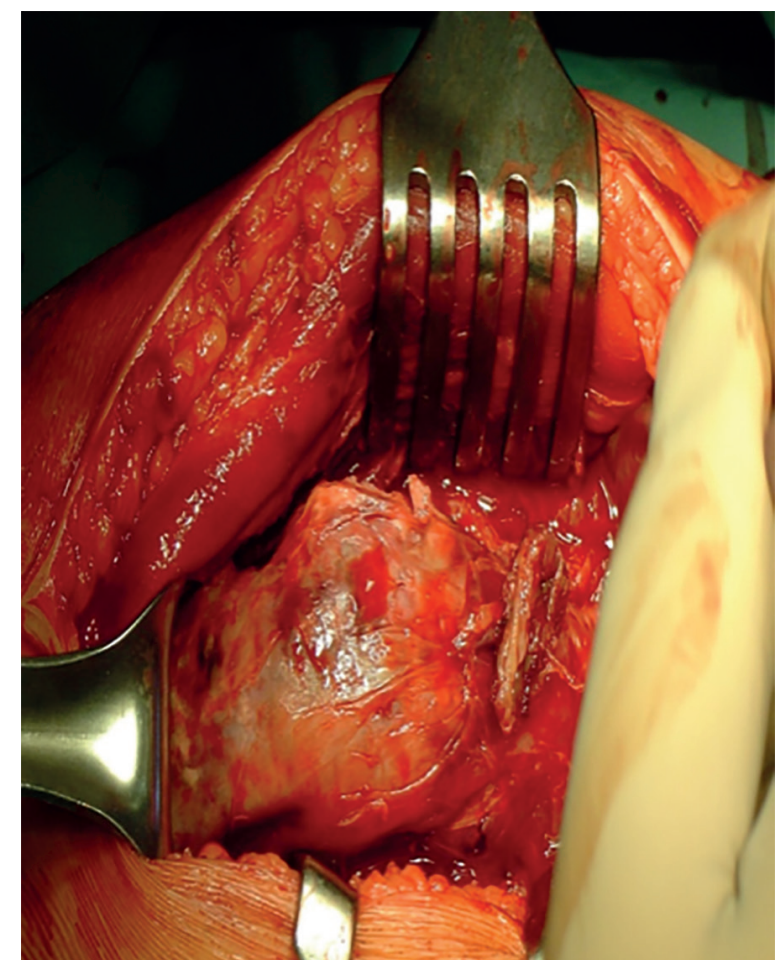

Figure 3. Intraoperative image of the part closer to the left femur

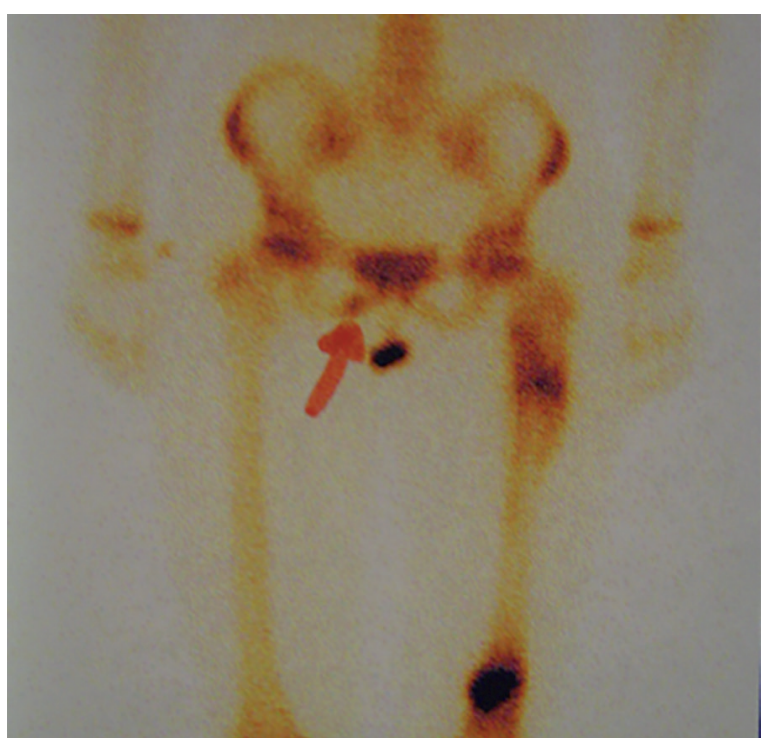

Figure 4. Scintigraphy of the skeleton showing hot areas in the right pubic bone and further parts of the femur

visible. One year after the surgery, the patient returned to full active and passive mobility and range of mobility. She did not feel any pain, either. Changes in the $X$-ray image of the right pubic bone and left femoral bone remitted spontaneously. 10 years after the diagnosis of the aneurysmal bone cyst, no recurrence or other ailments were observed (Fig. 6). Currently, the patient is physically active and practices sports.

\section{Discussion}

The strategy of treating aneurysmal bone cyst has been discussed by orthopaedic specialists treating bone tumors. The 


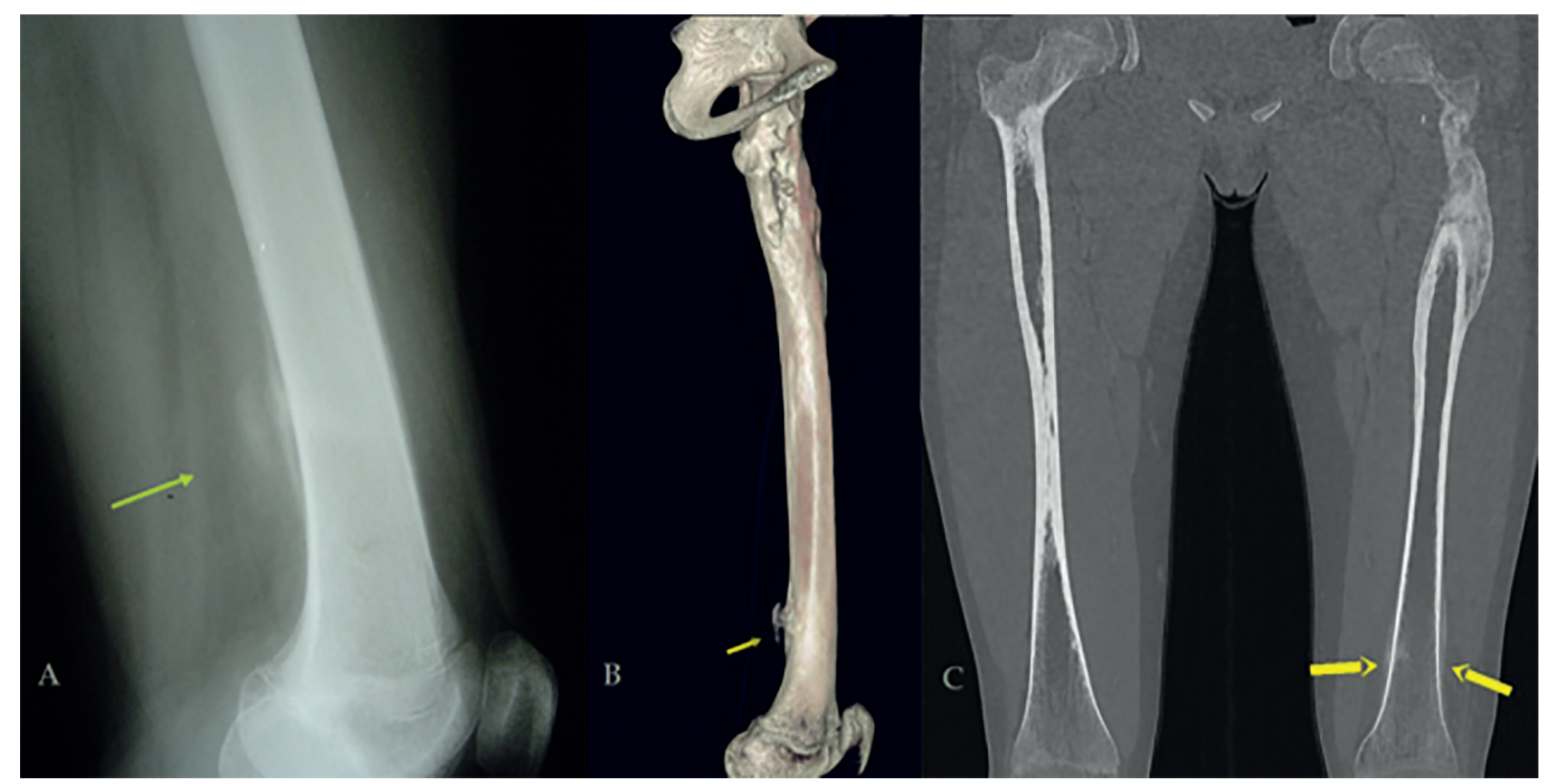

Figure 5. A - X-ray of the distal femur with the current lesion; B - Computed tomography with 3D reconstruction; C - Image of bone lesion in CT sections

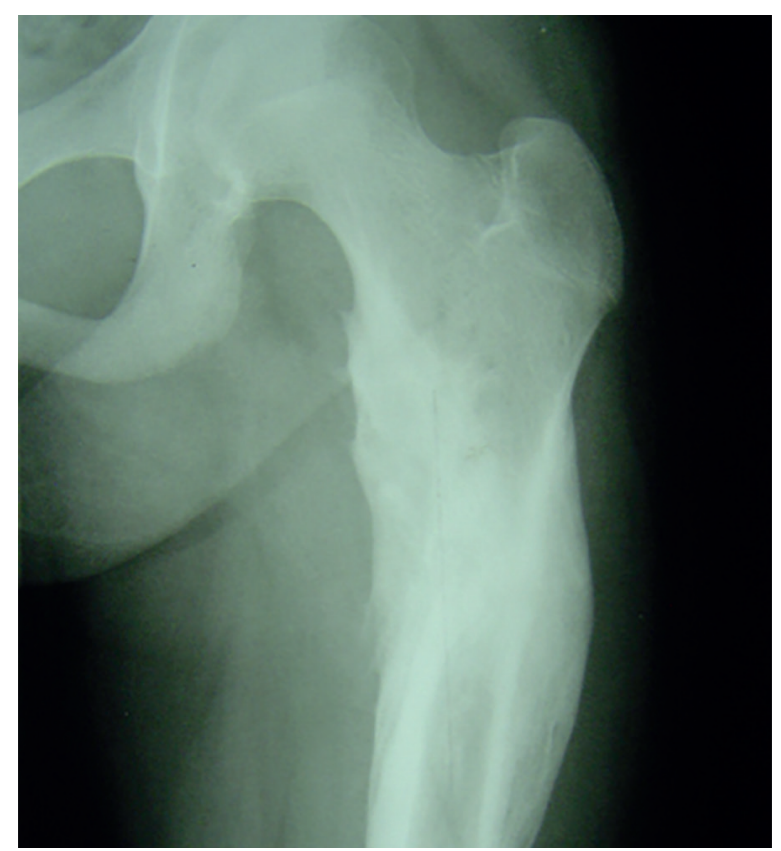

Figure 6. X-ray image 10 years after the surgery with visible remodeling in place of the removed bone cyst

treatment should be chosen individually depending on the location, extent and malignancy of the tumor [2]. However, despite many treatment techniques, between 5 and $40 \%$ of relapses are described [3].

Curettage and bone block excision are the ways to treat easily accessible lesions. However, in the treatment of difficult-to-reach ABCs [4-8] cryotherapy, percutaneous injections with preparations containing polydocanol (used, among others, in varicose veins sclerotization) or steroid preparations (methyl- prednisolone acetate, DepoMedrol) are applied. On the other hand, after resections of large ABC-type lesions, allogeneic bone grafts play an important role in the treatment of $A B C$-type lesions. However, they have a slower remodeling rate than autogenous grafts [9]. The cause of slower allograft remodeling is lack of active osteocytes in the transplant and lower degree of immune response [10].

Although $A B C$ is a benign tumor, the patient needs constant monitoring after the initial treatment. This is important because $A B C$ can be transformed into a malignant tumor, such as telangiectatic osteosarcoma or fibroblastic osteosarcoma [11-13]. Fortunately, these transformations are rare and are usually associated with earlier radiotherapy - the literature describes few cases of telangiectatic osteosarcoma after treatment of $A B C$ without prior irradiation.

In the case of the described patient, 10 years after the original treatment, despite the initially large extent and features of local malignancy, no relapse was observed.

\section{Conflict of interest: none declared}

\section{Andrzej Borowski}

Medical University of Lodz

Clinic of Orthopaedics and Paediatric Orthopaedics

ul. Pomorska 251

90-001 Łódź, Poland

e-mail:aborowski@xl.wp.pl

\section{Received: 2 Jan 2019}

Accepted: 6 May 2019

\section{References}

1. Cottalorda J, Bourelle S. Modern concepts of primary aneurysmal bone cyst. Archives of Orthopaedic and Trauma Surgery 2007; 127: 105-114. 
2. Guven M, Demirel M, Ozler T et al. An aggressive aneurysmal bone cyst of the proximal humerus and related complications in a pediatric patient. Strategies Trauma Limb Reconstr 2012; 7: 51-56.

3. Szendroi M, Cser I, Konya A et al. Aneurysmal bone cyst. A review of 52 primary and 16 secondary cases. Arch Orthop Trauma Surg 1992; 111: 318-322.

4. Campanacci M, Capanna R, Picci P. Unicameral and aneurysmal bone cysts. Clin Orthop Relat Res 1986; 25-36.

5. Chang CY, Kattapuram SV, Huang AJ et al. Treatment of aneurysmal bone cysts by percutaneous CT-guided injection of calcitonin and steroid. Skeletal Radiol 2017; 46: 35-40.

6. Brosjo O, Pechon P, Hesla A et al. Sclerotherapy with polidocanol for treatment of aneurysmal bone cysts. Acta Orthop 2013; 84: 502-505.

7. Varshney MK, Rastogi S, Khan SA et al. Is sclerotherapy better than intralesional excision for treating aneurysmal bone cysts? Clinical Orthopaedics and Related Research 2010; 468: 1649-1659.
8. Lampasi M, Magnani M, Donzelli O. Aneurysmal bone cysts of the distal fibula in children. Journal of Bone and Joint Surgery-British Volume 2007; 89b: 1356-1362.

9. Shih HN, Su JY, Hsu KY et al. Allogeneic cortical strut for benign lesions of the humerus in adolescents. J Pediatr Orthop 1997; 17: 433-436.

10. Glancy GL, Brugioni DJ, Eilert RE et al. Autograft versus allograft for benign lesions in children. Clin Orthop Relat Res 1991; 28-33.

11. Brindley GW, Greene JF, Jr., Frankel LS. Case reports: malignant transformation of aneurysmal bone cysts. Clin Orthop Relat Res 2005; 438: 282-287.

12. Kansagra AP, Wan JJ, Devulapalli KK et al. Malignant Transformation of an Aneurysmal Bone Cyst to Fibroblastic Osteosarcoma. Am J Orthop (Belle Mead NJ) 2016; 45: E367-E372.

13. Mascard E, Gomez-Brouchet A, Lambot K. Bone cysts: Unicameral and aneurysmal bone cyst. Orthopaedics \& Traumatology-Surgery \& Research 2015; 101: S119-S127. 